\title{
De la stabilité hydraulique des chambres d'équilibre dans certains cas complexes
}

\section{Surge tank stability in certain complex cases}

\author{
I'AR P. KAÏCHEV
}

INGÉNIEUR CIVIL, DIPLOMÉ DES SCIENGES TECHNIQUES, SOFIA ACADËMIE DES SGIENCES DE BULGARIE INSTITUT D'ÉNERGÉTIQUE

\begin{abstract}
Les amenagements hydrodectriques modernes. surtout dans les régions montagneuses, posent souvent plusieurs problèmes hydrauliques. Les questions touchant au régime non permanent forment une partie de ces problemes.

Dans ce mémoire, on a tenté de résoudre le problème de la stabilité hydraulique des chambres d'équilibre dans les divers cas complexes que l'ingénieur hydraulicien rencontre dans la pratique. On a étudié quelques systèmes d'alimenlation des centrales dont le canal en charge admet un débit supplémentaire, et aussi les chambres d'equilibre alimentées par deux relenues différentes. L'étude est basée sur les principes fondamentaux de la théorie des chambres d'équilibre.
\end{abstract}

\begin{abstract}
A number of hydranlic problems are often encountered in connection with modern hydroelectric schemes, especially in mountain areas. In this article the author attempts to solve the problem. of the hydraulic stability of surge. tanks in the various complex applications encountered in practice. The problems considered include power station supply systems, the supply canal of which has a second source of supply, and surge tanks supplied by two separate reservoirs. The article is based on the fundamental theory of surge tanks.
\end{abstract}

Considérons d'abord le système indiqué à la figure 1, alimenté par un réservoir au moyen d'un canal en charge, dans lequel se jette aussi un débit supplémentaire au moyen d'un autre canal en charge.

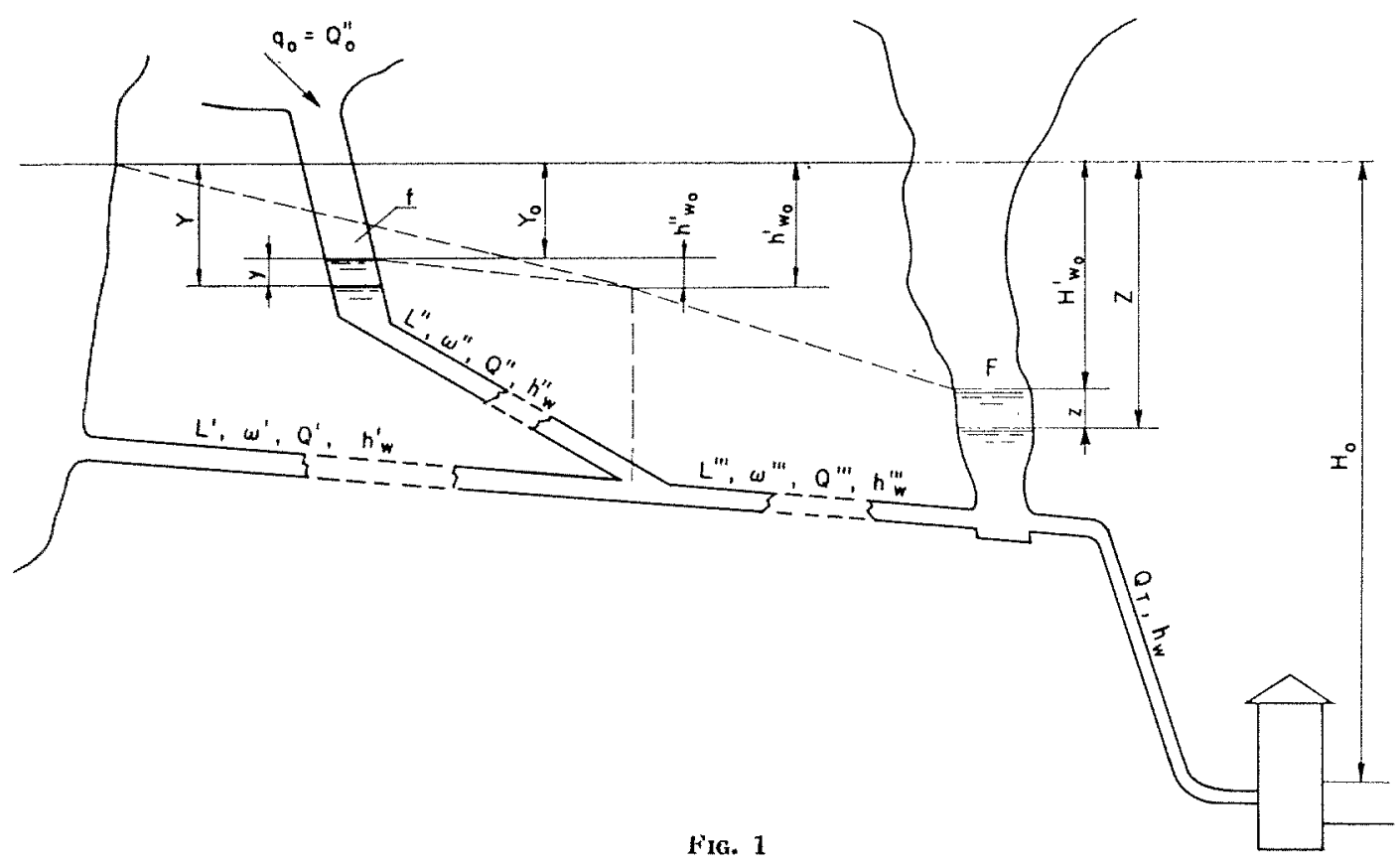


Nous devons remarquer que le débit supplémentaire se déverse dans un puits d'où part le deuxième canal en charge.

\section{NOTATIONS}

$L^{\prime}, L^{\prime \prime}, L^{\prime \prime \prime}=$ Longueurs des canaux en charge correspondants (v. la fig. 1); $\omega^{\prime}, \omega^{\prime \prime}, \omega^{\prime \prime \prime}=$ Seclions transversales des canaux en charge (*);

$$
\mathrm{L}_{r}^{\prime}=\frac{\mathrm{L}^{\prime}}{\omega^{\prime}} ; \quad \mathrm{L}_{r}^{\prime \prime}=\frac{\mathrm{L}^{\prime \prime}}{\omega^{\prime \prime}} ; \quad \mathrm{L}^{\prime \prime \prime}{ }_{r}=\frac{\mathrm{L}^{\prime \prime \prime}}{\omega^{\prime \prime \prime}}
$$

$Q^{\prime}, Q^{\prime \prime}, Q^{\prime \prime \prime}=$ Débits parcourant les canaux en charge;

$Q_{\mathrm{T}}=$ Débit utilisé par les turbines de la centrale hydro-électrique;

$q_{0}=$ Débit supplémentaire déversé dans le puits, et qui, pendant le régime non permanent, doit être considéré comme invariable;

$h^{\prime}=k^{\prime} \mathrm{Q}^{\prime 2} ; h^{\prime \prime}{ }_{w}=k^{\prime \prime} \mathrm{Q}^{\prime \prime 2} ; h^{\prime \prime \prime}{ }_{w}=l_{i}^{\prime \prime \prime} \mathrm{Q}^{\prime \prime \prime 2}$

$=$ Pertes de charge dans les canaux; ici $k^{(i)}$ sont les coefficients des pertes de charge exprimées par les débits correspondants:

$h_{w}-k \mathbf{Q}_{1}^{2}=$ Pertes de charge dans la conduite forcée $\left.{ }^{\star *}{ }^{\star}\right)$;

$\mathrm{H}_{w}^{\prime}=h^{\prime}+h^{\prime \prime \prime}{ }_{w} ; \mathrm{H}^{\prime \prime}{ }_{w}=h^{\prime \prime}{ }_{w}+h^{\prime \prime \prime}{ }_{w}$;

$\mathrm{H}_{0}=$ Chute brute de la centrale hydroélectrique, mesurée de la cote du plan d'eau dans la retenue;

$\mathbf{H}_{n}=$ Chute nette de l'usine;

Z - Ordonnée du plan d'eau dans la chambre d'équilibre par rapport à la cote du plan d'eau dans la retenue;

$\mathbf{Y}=$ Ordonnée du plan d'eau dans le puits, également par rapport à la cote du plan d'eau dans la retenue;

$\mathrm{F}=$ Section horizontale de la chambre d'équilibre au voisinage du plan d'eau à l'instant initial;

$f=$ Section horizontale du puits dans lequel se déverse le débit supplémentaire;

$\mathrm{N}=$ Puissance de l'usine hydroélectrique;

$\eta=$ Rendement des groupes installés;

$t=$ Temps;

$g=$ Accélération de la pesanteur.

Les autres symboles employés sont expliqués dans les passages correspondants du texte.

Plus loin, par l'indice « o 》apposé sous les notations précédentes, nous symboliserons leurs valeurs à l'instant initial. Les notations sans indice sont valables à chaque instant arbitraire $t$ pendant" le mouvement non permanent.

(") Si la dérivation en charge correspondante ren ferme quelques parties ayant des sections transversales différentes, il faut poser:

$$
I_{i}{ }^{(i)}=\sum_{L^{(i}} \frac{l_{k k}{ }^{(i)}}{\omega_{k}{ }^{(i)}}
$$

(*) Ici nous avons supposé que la centrale a une ou plusieurs conduites forcées identiques qui, à l'instant initial, sont chargées uniformément.
L'assurance d'avoir des phénomènes hydrauliques stables dans la chambre d'équilibre a une grande importance dans l'exploitation des centrales. Pour résoudre cette question dans les cas complexes considérés ici, nous avons adopté les mèmes simplifications que celles faites ordinairement dans la théorie des chambres d'équilibre, notamment :

$1^{\circ}$ On a négligé :

a) Les déformations élastiques des parois des canaux en charge soumis a une pression variable pendant le régime non permanent;

b) Les déformations élastiques de l'eau renfermée dans le système, soumise aussi à une pression variable; 
c) L'inertie de l'eau se trouvant dans la chambre d'équilibre et dans le puits et les pertes de charge pendant le mouvement du plan d'eau dans ces ouvrages;

d) L'influence possible de coup de bélier à l'instant initial ;

$2^{\circ}$ L'étude est effecluée en supposant que le régime non permanent s'établit sous la pression. atmosphérique identique partout où l'eau est à surface libre: la retenue, le puits, la chambre d'équilibre, le canal de fuite;

$3^{\circ}$ On a admis que le niveau de l'eau dans le canal d'évacuation ne change pas pendant le régime non permanent; pour les centrales ayant des turbines à réaction, c'est seulement approximativement vrai;

$4^{\circ}$ On a supposé aussi que le système de réglage des machines fonctionne « idéalement»; et qu'il maintient à chaque instant la puissance de la centrale en rapport avec la charge électrique.

En outre, l'étude est limitée aux « petits》 mouvements du plan d'eau dans la chambre d'équilibre et dans le puits, ainsi qu'on le fait ordinairement dans la théorie des chambres d'équilibre.

Supposons qu'à un instant donné l'usine hydroálectrique fonctionne avec une puissance $\mathrm{N}_{0}$. Si a cel instant arrive une petite variation, par exemple une augmentation de la charge de l'usine suivie d'une augmentation de la puissance ayant une petite valeur $\Delta \mathrm{N}=\mathrm{C}^{i e}$, cela conduira à une augmentation du débit appelé par les turbines avec la petite valeur $q_{\mathrm{T}}$. Il est ćvident que, par suite de l'inertie de l'eau dans les canaux en charge, $q_{\mathrm{T}}$ doit être prélevé entic̀rement et, plus tard, partiellement, sur la chambre d'équilibre; sans doute, cela provoquera dans la chambre d'équilibre un abaissement du plan d'eau ayant la petite valeur $z$.

Etant donné que $\mathrm{N}_{0}+\Delta \mathrm{N}=\mathrm{N}=\mathrm{C}^{\mathrm{te}}$, c'està-dire qu'après la variation, la puissance de la centrale reste invariable et correspondant à la charge électrique, nous pouvons écrire :

$$
\begin{aligned}
e \eta_{0} \mathrm{Q}_{\mathrm{T}_{\mathrm{o}}}\left(\mathrm{H}_{0}-\mathrm{H}_{w_{0}}^{\prime}-h_{w_{0}}\right)+\Delta \mathrm{N} \\
=e \eta_{\mathrm{i}} \mathrm{Q}_{\mathrm{Y}_{\mathrm{T}}}\left(\mathrm{H}_{0}-\mathrm{H}_{w_{0}}^{\prime}-z-h_{w}\right)
\end{aligned}
$$

Cependant :

$$
\begin{gathered}
\mathrm{Q}_{\mathrm{T}}==\mathrm{Q}_{\mathrm{T}_{0}}+q_{\mathrm{T}} \\
h_{w}=h_{w_{u}}\left(\frac{\mathrm{Q}_{\mathrm{T}_{\mathrm{v}}}+q_{\mathrm{T}}}{\mathrm{Q}_{\mathrm{T}_{\mathrm{v}}}}\right)^{2} \approx h_{w_{w}}+2 h_{w_{0}} \frac{q_{\mathrm{T}}}{\mathrm{Q}_{\mathrm{T}_{\mathrm{v}}}}
\end{gathered}
$$

et au lieu de (1) nous aurons :

$$
\begin{aligned}
& c r_{10} \mathrm{Q}_{\mathrm{T}_{\mathrm{o}}}\left(\mathrm{H}_{0}-\mathrm{H}_{w_{0}}^{\prime}-h_{w_{0}}\right)+\Delta \mathrm{N} \\
& =e \eta\left(\mathrm{Q}_{\mathrm{T}_{\mathrm{o}}}+q_{\mathrm{T}}\right)\left(\mathrm{H}_{0}-\mathrm{H}_{w_{0}}^{\prime}-z-\mathrm{h}_{w_{0}}-2 h_{w_{\mathrm{o}}} \frac{\eta_{\mathrm{T}}}{\mathrm{Q}_{\mathrm{T}_{\mathrm{o}}}}\right)
\end{aligned}
$$

En tenant compte de ce que, pendant les petits mouvements du plan d'eau dans la chambre d'équilibre, le rendement des groupes ne change pas considérablement, c'est-à-dire en posant que $\eta \approx \eta_{0}$ et en négligeant les petites valeurs d'ordre supérieur $q_{\mathrm{T}} z$ et $2 h_{w_{0}}\left(q_{\mathrm{r}^{2}}{ }^{2} / Q_{\mathrm{r}_{0}}\right)$, nous obtiendrons :

$$
q_{\mathrm{T}}=\frac{\mathrm{Q}_{\mathrm{T}_{\mathrm{n}}}}{\mathrm{H}_{1}} z+\mathrm{S}_{0}
$$

où les symboles supplémentaires $H_{1}$ et $S_{0}$ ont pour valeurs :

$$
\begin{gathered}
\mathrm{H}_{1}=\mathrm{H}_{0}-\mathrm{H}_{w_{0}}^{\prime}-3 h_{w_{0}} \\
\mathrm{~S}_{0}=\frac{\Delta \mathrm{N}}{e \mathrm{r}_{1}}
\end{gathered}
$$

L'équation différentielle fondamentale du régime non permanent et l'équation de continuité, écrites pour les trois parties du système d'amenée, nous donnent :

$$
\begin{gathered}
Z=h_{w}^{\prime}+h^{\prime \prime \prime}{ }_{w}+\frac{\mathrm{L}_{r} d \mathrm{Q}^{\prime}}{g}+\frac{\mathrm{L}^{\prime \prime \prime} r}{g} \frac{d \mathrm{Q}^{\prime \prime \prime}}{d t} \\
\mathrm{Y}+h^{\prime \prime}{ }_{i ;}+\frac{\mathrm{L}^{\prime \prime}{ }_{r}}{g} \frac{d \mathrm{Q}^{\prime \prime}}{d t}=h_{w}^{\prime}+\frac{\mathrm{L}_{r}^{\prime}}{g} \frac{d \mathrm{Q}^{\prime}}{d t} \\
\mathrm{Q}_{\mathrm{C}}=\mathrm{Q}^{\prime \prime \prime}+\mathrm{F} \frac{d Z}{d t} \\
\mathrm{Q}^{\prime \prime}=\mathrm{Q}^{\prime \prime}{ }_{0}+t \frac{d \mathrm{Y}}{d t}=q_{0}+f \frac{d \mathrm{Y}}{d t}
\end{gathered}
$$

Le plan d'ean dans le puits subira aussi une variation ayant la petite valeur $y$. Les débits parcourant les canaux en charge subiront des variations correspondantes ayant les petites valeurs $q^{\prime}, q^{\prime \prime}, q^{\prime \prime \prime}=q^{\prime}+q^{\prime \prime}$. Alors on peut écrire :

$$
\begin{aligned}
Z & =\mathrm{H}_{w_{0}}^{\prime}+z=h_{w_{0}}+h_{w_{0}}^{\prime \prime}+z\left(^{\star}\right) \\
\mathrm{Y} & =\mathrm{Y}_{0}+y=h_{w_{0}}^{\prime}-h_{w_{0}}^{\prime \prime}+y \\
\mathrm{Q}^{\prime} & =\mathrm{Q}_{0}^{\prime}+q^{\prime} \\
\mathrm{Q}^{\prime \prime} & =\mathrm{Q}_{0}^{\prime \prime}+q^{\prime \prime} \\
\mathrm{Q}^{\prime \prime \prime} & =\mathrm{Q}^{\prime \prime \prime}+q^{\prime \prime \prime}=\mathrm{Q}_{0}^{\prime}+\mathrm{Q}_{0}^{\prime \prime}+q^{\prime}+q^{\prime \prime}
\end{aligned}
$$

Des équations (1) et (5) nous obtiendrons :

$$
\frac{d z}{d l}-\frac{\mathrm{Q}_{\mathrm{L}_{0}}}{\mathrm{H}_{1} \mathrm{~F}} z+\frac{1}{\mathrm{~F}} q^{\prime}+\frac{1}{\mathrm{~F}} q^{\prime \prime}=\frac{\mathrm{S}_{0}}{\mathrm{~F}}
$$

(*) Si la chambre d'équilibre comporte un étranglement, aux pertes de charge, dans l'équation du nouvement non permanent, if faut ajouter la valeur $k_{\text {e }}[F(d z / d t)]^{2}$. On voit que cette valeur est petite, du deuxième ordre par rapport à $(d z / d t)$, et qu'on peut la négliger. 
et au lieu de l'équation (6) :

$$
\frac{d y}{d t}-\frac{1}{f} q^{\prime \prime}=0
$$

Après certaines transformations et en négligeant les termes contenant les valeurs $q^{\prime 2}$ et $q^{\prime \prime 2}$, comme étant petites, d'ordre supérieur, les écqualions (3) et (4) donnent :

$$
\begin{aligned}
& \frac{d q^{\prime}}{d t}-\frac{g \mathrm{~L}_{r}^{\prime \prime}}{\mathrm{L}_{r}^{\prime} \mathrm{L}^{\prime \prime \prime}{ }_{r}+\mathrm{L}_{r}^{\prime \prime}\left(\mathrm{L}_{r}^{\prime}+\mathrm{L}^{\prime \prime \prime}{ }_{r}\right)} z- \\
& -\frac{g \mathbf{L}^{\prime \prime \prime}{ }_{r}}{\mathrm{~L}_{r}^{\prime} \mathrm{L}^{\prime \prime \prime}{ }_{r}+\mathrm{L}^{\prime \prime}{ }_{r}\left(\mathrm{~L}_{r}{ }_{r}+\mathrm{L}^{\prime \prime \prime}{ }_{r}\right)} y+ \\
& +\frac{2 g\left[k^{\prime} \mathrm{Q}_{0}^{\prime} \mathrm{L}^{\prime \prime \prime}{ }_{r}+\left(k^{\prime} \mathrm{Q}_{0}^{\prime}+k^{\prime \prime \prime} \mathrm{Q}^{\prime \prime \prime}{ }_{0}\right) \mathrm{L}^{\prime \prime}{ }_{r}\right]}{\mathrm{L}_{r}^{\prime} \mathrm{L}^{\prime \prime \prime}{ }_{r}+\mathrm{L}^{\prime \prime}{ }_{r}\left(\mathrm{~L}_{r}^{\prime}+\mathrm{L}^{\prime \prime \prime}{ }_{r}\right)} q^{\prime} \\
& -\frac{2 g\left[k^{\prime \prime} \mathrm{Q}_{0}^{\prime \prime} \mathrm{L}^{\prime \prime \prime}{ }_{r}-k^{\prime \prime \prime} \mathrm{Q}^{\prime \prime \prime}{ }_{0} \mathrm{~L}^{\prime \prime}{ }_{r}\right]}{\mathrm{L}_{r} \mathrm{~L}^{\prime \prime \prime}{ }_{r}+\mathrm{L}^{\prime \prime}{ }_{r}\left(\mathrm{~L}_{r}+\mathrm{L}^{\prime \prime \prime}{ }_{r}\right)} q^{\prime \prime}=0
\end{aligned}
$$

$$
\begin{aligned}
& \frac{d q^{\prime \prime}}{d t}-\frac{g \mathrm{~L}_{r}^{\prime}}{\mathrm{L}_{r}^{\prime} \mathrm{L}^{\prime \prime \prime}{ }_{r}+\mathrm{L}_{r}^{\prime \prime}\left(\mathrm{L}_{r}^{\prime}+\mathrm{L}^{\prime \prime \prime}{ }_{r}\right)} z+ \\
& +\frac{g^{\prime}\left(\mathbf{L}_{r}^{\prime}+\mathbf{L}^{\prime \prime \prime}{ }_{r}\right)}{\mathrm{L}_{r}{ }^{\prime} \mathrm{L}^{\prime \prime \prime}{ }_{r}+\mathbf{L}_{r}^{\prime \prime}\left(\mathbf{L}_{r}+\mathbf{L}^{\prime \prime \prime}{ }_{r}\right)} y- \\
& -\frac{2 g\left[k^{\prime} \mathrm{Q}_{0}^{\prime} \mathrm{L}^{\prime \prime \prime}{ }_{r}-k^{\prime \prime \prime} \mathrm{Q}^{\prime \prime \prime}{ }_{0} \mathrm{~L}_{r}\right]}{\mathrm{L}_{r}^{\prime} \mathrm{L}^{\prime \prime \prime}{ }_{r}+\mathrm{L}^{\prime \prime}{ }_{r}\left(\mathrm{~L}_{r}^{\prime}+\mathrm{L}^{\prime \prime \prime}{ }_{r}\right)} q^{\prime}+ \\
& +\frac{2 g\left[k^{\prime \prime} \mathrm{Q}^{\prime \prime}{ }_{0} \mathrm{~L}^{\prime \prime \prime}{ }_{r}+\left(k^{\prime \prime} \mathrm{Q}^{\prime \prime}{ }_{0}+k^{\prime \prime \prime} \mathrm{Q}^{\prime \prime \prime}{ }_{0}\right) \mathrm{L}_{r}^{\prime}\right]}{\mathrm{L}_{r}^{\prime} \mathrm{L}^{\prime \prime \prime}{ }_{r}+\mathrm{L}_{r}^{\prime \prime}{ }_{r}\left(\mathrm{~L}_{r}^{\prime}+\mathrm{L}^{\prime \prime \prime}{ }_{r}\right)} q^{\prime \prime}=0
\end{aligned}
$$

Les équations (7), (8), (9) et (10) représentent un système linéaire d'équations différentielles avec des coefficients constants, qui a la forme générale suivante :

$$
\left.\begin{array}{l}
\frac{d z}{d t}+a_{11} z+a_{12} y+a_{13} q^{\prime}+a_{14} q^{\prime \prime}=\mathrm{S}_{0} / \mathrm{F} \\
\frac{d y}{d t}+a_{21} z+a_{22} y+a_{23} q^{\prime}+a_{24} q^{\prime \prime}=0 \\
\frac{d q^{\prime}}{d t}+a_{31} z+a_{32} y+a_{33} q^{\prime}+a_{34} q^{\prime \prime}=0 \\
\frac{d q^{\prime \prime}}{d t}+a_{41} z+a_{42} y+a_{43} q^{\prime}+a_{44} q^{\prime \prime}=0
\end{array}\right\}
$$

Ici :

$$
\begin{aligned}
& a_{11}=-\frac{\mathrm{Q}_{\mathrm{I}_{\mathrm{a}}}}{\mathrm{H}_{1} \mathrm{~F}} ; \quad a_{12}=0 ; \quad a_{13}=1 / \mathrm{F} ; a_{14}=1 / \mathrm{F} ; \\
& a_{21}=0 ; \quad a_{22}=0 ; \quad a_{23}=0 ; \quad a_{24}=-1 / f
\end{aligned}
$$

$$
\begin{aligned}
& a_{31}=-\frac{g \mathrm{~L}^{\prime \prime}{ }_{r}}{\mathrm{~L}_{r}^{\prime} \mathrm{L}^{\prime \prime \prime}{ }_{r}+\mathrm{L}^{\prime \prime}{ }_{r}\left(\mathrm{~L}_{r}^{\prime}+\mathrm{L}^{\prime \prime \prime}{ }_{2}\right)} \\
& a_{32}=-\frac{g \mathrm{~L}^{\prime \prime \prime}{ }_{r}}{\mathrm{~L}_{r}^{\prime} \mathrm{L}^{\prime \prime \prime}{ }_{r}+\mathrm{I}^{\prime \prime}{ }_{r}\left(\mathrm{~L}_{r}^{\prime}+\mathrm{L}^{\prime \prime \prime}{ }_{r}\right)} \\
& a_{33}=\frac{2 g\left[k^{\prime} \mathrm{Q}_{0}^{\prime} \mathrm{L}^{\prime \prime \prime}{ }_{r}+\left(k^{\prime} \mathrm{Q}_{0}^{\prime}+k^{\prime \prime \prime} \mathrm{Q}^{\prime \prime \prime}{ }_{0}\right) \mathrm{L}^{\prime \prime}{ }_{r}\right]}{\mathrm{L}_{r}^{\prime} \mathrm{L}^{\prime \prime \prime}{ }_{r}+\mathrm{L}^{\prime \prime}{ }_{r}\left(\mathrm{~L}_{r}^{\prime}+\mathrm{L}^{\prime \prime \prime}{ }_{r}\right)} \\
& a_{34}=-\frac{2 g\left[k^{\prime \prime} \mathrm{Q}^{\prime \prime}{ }_{0} \mathrm{~L}^{\prime \prime \prime}{ }_{r}-k^{\prime \prime \prime} \mathrm{Q}^{\prime \prime \prime}{ }_{0} \mathrm{~L}^{\prime \prime}{ }_{r}\right]}{\mathrm{L}_{r}^{\prime} \mathrm{L}^{\prime \prime \prime}{ }_{r}+\mathrm{L}^{\prime \prime}{ }_{r}\left(\mathrm{~L}_{r}^{\prime}+\mathrm{L}^{\prime \prime \prime}{ }_{r}\right)} \\
& a_{41}=-\frac{g \mathrm{~L}_{r}^{\prime}}{\mathrm{L}_{r}^{\prime} \mathrm{L}^{\prime \prime \prime}{ }_{r}+\mathrm{L}_{r}^{\prime \prime}\left(\mathrm{L}_{\gamma}^{\prime}+\mathrm{L}^{\prime \prime \prime}{ }_{r}\right)} \\
& a_{42}=\frac{g\left(\mathrm{~L}_{r}^{\prime}+\mathrm{L}^{\prime \prime \prime}{ }_{r}\right)}{\mathrm{L}_{r}^{\prime} \mathrm{L}^{\prime \prime \prime}{ }_{r}+\mathrm{L}^{\prime \prime}{ }_{r}\left(\mathrm{~L}_{r}^{\prime}+\mathrm{L}^{\prime \prime \prime}{ }_{r}\right)} \\
& a_{43}=-\frac{2 g\left[k^{\prime} \mathbf{Q}_{0}^{\prime} \mathrm{L}^{\prime \prime \prime}{ }_{r}-k^{\prime \prime \prime} \mathrm{Q}^{\prime \prime \prime}{ }_{0} \mathrm{~L}_{r}\right]}{\mathrm{I}^{\prime} \mathrm{L}^{\prime \prime \prime}{ }_{r}+\mathrm{L}^{\prime \prime}{ }_{r}\left(\mathrm{~L}^{\prime}{ }_{r}+\mathrm{L}^{\prime \prime}{ }_{r}\right)} \\
& a_{44}=\frac{2 g\left[k^{\prime \prime} \mathrm{Q}_{0}^{\prime \prime} \mathrm{L}^{\prime \prime \prime}{ }_{r}+\left(k^{\prime \prime} \mathrm{Q}^{\prime \prime}{ }_{0}+k^{\prime \prime \prime} \mathrm{Q}^{\prime \prime{ }_{0}}\right) \mathrm{L}_{r}^{\prime}\right]}{\mathrm{L}_{r}^{\prime} \mathrm{L}^{\prime \prime \prime}{ }_{r}+\mathrm{L}^{\prime \prime}{ }_{r}\left(\mathrm{~L}^{\prime}{ }_{r}+\mathrm{L}^{\prime \prime \prime}{ }_{r}\right)}
\end{aligned}
$$

Il n'est pas difficile de voir que la résolution du système (11) conduit à la résolution du système homogène correspondant avec l'exactitude d'une valeur constante, c'est-à-dire :

$$
\begin{gathered}
z=z_{p}+z_{h} \\
y=y_{p}+y_{h} \\
q^{\prime}=q_{p}^{\prime}+q_{h}^{\prime} \\
q^{\prime \prime}=q_{p}^{\prime \prime}+q_{h}^{\prime \prime}
\end{gathered}
$$

ò̀ :

$$
\begin{aligned}
z_{p} & =y_{p}=\mathrm{C}^{\mathrm{te}} \\
q_{p}^{\prime} & =\mathrm{C}^{\mathrm{te}} \\
q_{p}^{\prime \prime} & =0
\end{aligned}
$$

et $z_{h}, y_{h}, q_{h}^{\prime}, q^{\prime \prime}{ }_{h}$ sont les valeurs correspondantes obtenues en résolvant le système homogène des équations différentielles, notamment :

$$
\begin{aligned}
z_{h} & =\sum_{1}^{4} \mathrm{C}_{i}^{\prime} e^{r_{i}} \\
y_{h} & =\sum_{1}^{4} \mathrm{C}_{i}^{\prime \prime} e^{r_{i} t} \\
q^{\prime}{ }_{h} & =\sum_{1}^{4} \mathrm{D}_{i}^{\prime} e^{r_{i} t} \\
q^{\prime \prime}{ }_{h} & =\sum_{1}^{4} \mathrm{D}_{i}^{\prime \prime} e^{r_{i} t}
\end{aligned}
$$


Ici $e$ est la base des logarithmes naturels et $r_{i}$ sont les racines de l'équalion caractéristique, qui peut être écrite sous la forme suivante :

$\left|\begin{array}{llll}a_{11}+r & 0 & a_{13} & a_{14} \\ 0 & r & 0 & a_{24} \\ a_{31} & a_{32} & a_{33}+r & a_{34} \\ a_{41} & a_{42} & a_{43} & a_{44}+r\end{array}\right|=0$

En résolvant (14) envers $r$, on obtient une équation algébrique du quatrième degré :

$$
\mathrm{A}_{0} r^{4}+\mathrm{A}_{1} r^{3}+\mathrm{A}_{2} r^{2}+\mathrm{A}_{3} r^{2}+\mathrm{A}_{4}=0
$$

Les coefficients $A_{j}$ ont les valeurs suivantes:

$$
\begin{aligned}
& A_{0}=\frac{\mathrm{L}_{r}^{\prime} \mathrm{L}^{\prime \prime \prime}{ }_{r}+\mathrm{L}^{\prime \prime}{ }_{r}\left(\mathrm{~L}_{r}^{\prime}+\mathrm{L}^{\prime \prime \prime}{ }_{r}\right)}{g} \\
& \mathrm{~A}_{1}=-\frac{\mathrm{Q}_{\mathrm{T}_{0}}}{g \mathrm{H}_{1} \mathrm{~F}^{\prime \prime}}\left[\mathrm{L}^{\prime} \mathrm{L}^{\prime \prime \prime}{ }_{r}+\mathrm{L}^{\prime \prime}{ }_{r}\left(\mathrm{~L}^{\prime}{ }_{r}+\mathrm{L}^{\prime \prime \prime}{ }_{r}\right)\right]+ \\
& +2\left[k^{\prime} Q_{0}^{\prime}\left(\mathrm{L}^{\prime \prime}{ }_{r}+\mathrm{L}^{\prime \prime \prime}{ }_{r}\right)\right]+k^{\prime \prime} \mathrm{Q}^{\prime \prime}{ }_{0}\left(\mathrm{~L}_{r}^{\prime}+\mathrm{L}^{\prime \prime \prime}{ }_{r}\right)+ \\
& \left.+k^{\prime \prime \prime} \mathrm{Q}^{\prime \prime \prime}{ }_{0}\left(\mathrm{~L}_{r}^{\prime}+\mathrm{L}^{\prime \prime}{ }_{r}\right)\right] \\
& \mathrm{A}_{2}=-\frac{2 \mathrm{Q}_{\mathrm{T}_{0}}}{\mathrm{H}_{1} \mathrm{~F}}\left[\boldsymbol{K}^{\prime} \mathrm{Q}_{0}^{\prime}\left(\mathrm{L}^{\prime \prime}{ }_{r}+\mathrm{L}^{\prime \prime \prime}{ }_{r}\right)+\right. \\
& \left.+k^{\prime \prime} \mathrm{Q}^{\prime \prime}{ }_{0}\left(\mathrm{~L}_{r}^{\prime}+\mathrm{L}^{\prime \prime \prime}{ }_{r}\right)+k^{\prime \prime \prime} \mathrm{Q}^{\prime \prime \prime}{ }_{0}\left(\mathrm{~L}_{r}^{\prime}+\mathrm{L}^{\prime \prime}{ }_{r}\right)\right]+ \\
& +4 g\left(k^{\prime} \mathrm{Q}_{0}^{\prime} k^{\prime \prime} \mathrm{Q}_{0}^{\prime \prime}+k^{\prime} \mathrm{Q}_{0}^{\prime} k^{\prime \prime \prime} \mathrm{Q}^{\prime \prime \prime}{ }_{0}+\right. \\
& \left.+k^{\prime \prime} \mathrm{Q}^{\prime \prime}{ }_{0} k^{\prime \prime \prime} \mathrm{Q}^{\prime \prime \prime}{ }_{0}\right)+\frac{1}{f}\left(\mathrm{~L}_{r}+\mathrm{L}^{\prime \prime \prime}{ }_{r}\right)+ \\
& +\frac{1}{\mathrm{~F}}\left(\mathrm{~L}^{\prime} \cdot+\mathrm{L}^{\prime \prime}\right)
\end{aligned}
$$$$
\mathrm{A}_{3}=-4 g \underset{\mathrm{H}_{1} \mathrm{~F}}{\mathrm{Q}_{\mathrm{C}_{0}}}\left[k^{\prime} \mathrm{Q}_{0}{ }_{0} k^{\prime \prime} \mathrm{Q}_{0}^{\prime \prime}+k^{\prime} \mathrm{Q}_{0}^{\prime} k^{\prime \prime \prime} \mathrm{Q}^{\prime \prime \prime}{ }_{0}+\right.
$$$$
\left.\left.+k^{\prime \prime} \mathrm{Q}_{0}^{\prime \prime} k^{\prime \prime \prime} \mathrm{Q}^{\prime \prime \prime}{ }_{0}\right)+\frac{\mathrm{L}_{r}^{\prime}+\mathrm{L}^{\prime \prime \prime}{ }^{\prime}}{4 g f}\right]+
$$$$
+\frac{2 g}{f}\left(k^{\prime} \mathrm{Q}_{0}^{\prime}+k^{\prime \prime \prime} \mathrm{Q}_{0}^{\prime \prime \prime}\right)+-\frac{2 g}{\mathrm{~F}}\left(k^{\prime} \mathrm{Q}_{0}^{\prime}+k^{\prime \prime} \mathrm{Q}^{\prime \prime}{ }_{11}\right)
$$$$
\Lambda_{1}=-\frac{g}{f \mathrm{~F}}\left|1-\begin{array}{c}
2 \mathrm{Q}_{\mathrm{Y}^{\prime \prime}} \\
\mathrm{H}_{1}
\end{array}\left(k^{\prime} \mathrm{Q}^{\prime}{ }_{0}+k^{\prime \prime \prime} \mathrm{Q}^{\prime \prime \prime}{ }_{0}\right)\right|
$$

Exprimés en fonction de $F$, les coefficients de l'équation caractéristique ont, en général, Ja forme :

$$
\begin{aligned}
& A_{0}=\beta_{0} \\
& A_{1}=(1 / F) \alpha_{1}+\beta_{1} \\
& A_{2}=(1 / F) \alpha_{2}+\beta_{2} \\
& A_{3}=(1 / F) \alpha_{3}+\beta_{3} \\
& A_{4}=(1 / F) \alpha_{4}
\end{aligned}
$$

Il est évident que les valeurs constantes des intégrales particulières $z_{p}, y_{p}, q_{p}^{\prime}$ et $q_{p}^{\prime \prime}$ n'ont aucune signification pour la stabilité des phénomènes hydrauliques dans la chambre d'équilibre. En outre, les équations (13) montrent que les fonctions $z_{h}, y_{h}, q_{h}^{\prime}$ et $q^{\prime \prime}{ }_{h}$ et par conséquent les fonctions $z, y, q^{\prime}$ et $q^{\prime \prime}$ ne tendront dans le temps vers des valeurs définies que dans le cas où les racines de l'équation caractéristique (15) ne sont pas positives, ou bien n'ont pas de parties réelles positives si elles sont complexes.

Comme on le sait d'après la théorie des équations algébriques, pour que les racines $r_{i}$ soient négatives ou pour qu'elles n'aient pas de parties réelles positives si elles sont complexes, il est nécessaire et suffisant, pour $A_{0}>0$ (ce qui peut toujours être obtenu), d'observer les conditions suivantes (théorème de Hurvitz):

$$
\begin{aligned}
A_{1} & >0 \\
A_{2} & >0 \\
A_{3} & >0 \\
A_{4} & >0 \\
A_{1} A_{2} A_{3}-A_{0} A_{3}{ }^{2} & -A_{1}{ }^{2} A_{4}>0
\end{aligned}
$$

ou respectivement :

$$
\begin{aligned}
& \mathrm{F}>-\left(\alpha_{1} / \beta_{1}\right) \\
& \mathrm{F}>-\left(\alpha_{2} / \beta_{2}\right) \\
& \mathrm{F}>-\left(\alpha_{3} / \beta_{3}\right) \\
& \alpha_{4}>0 \\
& \mu_{0} \mathrm{~F}^{3}+\mu_{1} \mathrm{~F}^{2}+\mu_{2} \mathrm{~F}+\mu_{3}>0
\end{aligned}
$$

On voit, par conséquent, que les conditions (16), (17), (18) et (20) exigent que la chambre d'équilibre ait une section horizontale minimale qui doit être plus grande d'une valeur critique définie; la condition (19) conduit à la limitation des pertes de charge.

Voyons quelles sont les limitations exigeant les conditions (16), (17) et (18).

Supposons que nous ayons choisi une valeur de la section $\mathrm{F}$, telle que les conditions (16), (17) et (18) soient observées, mais de telle manière qu'un de ces coefficients restant positif, tende vers zéro. Soit, par exemple, $A_{1}$ ce coefficient. Alors, nous aurons :

$$
\begin{aligned}
& A_{1} \rightarrow 0 \text { (mais restant positif) } \\
& A_{2}>0 \\
& A_{3}>0
\end{aligned}
$$

et aussi $A_{4}>0$. 
On voit facilement que, dans ce cas, la condilion (20) n'est pas observée.

Nous aurons les mêmes résultats si l'un quelconque des autres coefficients, ou si deux ou les trois coefficients restant positifs tendent vers zéro.

Par suite, la condition (20) contient les conditions (16), (17) et (18) et si elle est observée, cela indique que les conditions précédentes sont cigalement observées.

Résolvant l'inégalité (20), respectivement (20 a), nous obtenons :

$$
\mathbf{F}^{\prime}>\mathbf{F}_{c *}
$$

où $F_{t r}$ sera la plus grande racine positive de l'éfuation obtenue en égalant à zéro la partie gauche de l'inégalité $(20 a)$.

Evidemment, l'inégalité (21) doit être observée pour toutes les situations possibles du plan d'eau dans la retenue et dans tous les cas possibles par rapport au gradient de charge de la centrale.

Nous devons remarquer que $\mathrm{F}_{r r}$ sera à un cerlain degré fonction de $f$, et qu'à chaque valeur de $f$ correspondra une valeur définie de F. Cependant $f$ dépend avant tout de la construction du puits qui est éloigné de la chambre d'équilibre et l'assurance d'avoir des phénomènes stables en augmentant $f$ n'est pas économiquement justifiée. C'est pourquoi la stabilité hydratulique doit être assurée en choisissant une valeur convenable pour la section horizontale de la chambre déquilibre.

Ën raison du caractère bien compliqué des coefficients $a_{i k}$ et $\mathrm{A}_{i}$, nous pensons qu'il serait déplacé de chercher l'expression générale pour Far, parce qu'on ne peut pas en attendre une simplification sérieuse des calculs. C'est pourquoi l'inégalité $(20 a)$ et l'équation correspondante peuvent être résolues en calculant les valeurs particulières de $u_{i}$, ce qui rend plus loin les calculs plus simples et plus clairs.

Du fait que les déductions précédentes sont obtenues en laisant des suppositions simplificatrices, dans la pratique, on choisit la valeur de $F_{\text {min }}$ en augmentant la raleur de $F_{t r}$, par exemple avec $20 \div 50 \%$, c'est-à-dire :

$$
\mathrm{F}_{\text {min }}=(1,2 \div 1,5) \mathrm{F}_{\mathrm{r}, r}
$$

Ia condition $A_{4}>0$, après certaines translormations, donne :

$$
\mathrm{H}^{\prime} w_{0}+h_{100}<\frac{\mathrm{H}_{0}-2 k^{\prime} \mathrm{Q}_{0}^{\prime} q_{0}}{3}
$$

qui nous montre la valeur des pertes de charge qui ne doit pas être dépassée pour que les mou- vements du plan d'eau dans la chambre d'équilibre soient stables.

Montrons le sens de celle condition qui, comme on le voit d'après (23), se rapporte au régime permanent dans le système.

Soit la puissance de l'usine à un instant :

$$
\mathbf{N}=e r_{j} \mathrm{Q}_{\Gamma}\left(\mathrm{H}_{0}-{ }^{-} \mathrm{H}^{\prime}{ }_{w}-h_{w}\right)
$$

Il est évident que la variation de la puissance peut être obtenue seulement en modifiant le débit appelé par les turbines. Supposons que le rendement ne varie pas, c'est-à-dire $\eta=C^{\text {te }}$ et aussi que les turbines peuvent utiliser des débits arbitraires. Cherchons les conditions pour que la puissance de l'usine soit maximale. Pour cela, il faut :

$\frac{d \mathrm{~N}}{d \mathrm{Q}_{\mathrm{T}}}=e \tau_{1} \frac{d\left\{\mathrm{Q}_{\mathrm{T}}\left[\mathrm{H}_{0}-k^{\prime} \mathrm{Q}^{2}-k^{\prime \prime \prime}\left(\mathrm{Q}^{\prime}+q_{0}\right)^{2}-k Q_{\mathrm{T}}{ }^{2}\right]\right\}}{d \mathrm{Q}_{\mathrm{T}}}=c$

En observant que $Q_{\mathrm{T}}=\left(Q^{\prime}+q_{0}\right)$ et $d Q_{\mathrm{T}}=d Q^{\prime}$ on obtiendra :

$$
\mathrm{H}^{\prime}{ }_{w}+h_{w}=\frac{\mathrm{H}_{0}-2 k^{\prime} \mathrm{Q}^{\prime} q_{0}}{3}
$$

Cela signifie qu'à la puissance maximale de l'usine hydroélectrique correspondent un débit et des pertes de charge satisfaisant à la condition (24) qui représente les deux parties égalisées de l'inégalité (23). Exprimée graphiquement, la dépendance entre la puissance et le débit avec lequel fonctionne la centrale est montrée par la figure 2.

On voit, d'après la figure 2, que la puissance maximale est atteinte quand la centrale fonctionne avec un débit $Q_{\mathrm{T}}=\mathrm{Q}_{m}^{\prime}+q_{0}$. Toute augmentation de débit en dehor's de cette valeur conduit à une diminution de la puissance, au lieu d'une augmentation. Le système de réglage des machines continuera à fonctionner avec une tendance à maintenir la puissance en rapport avec la charge électrique. Une nouvelle augmentation

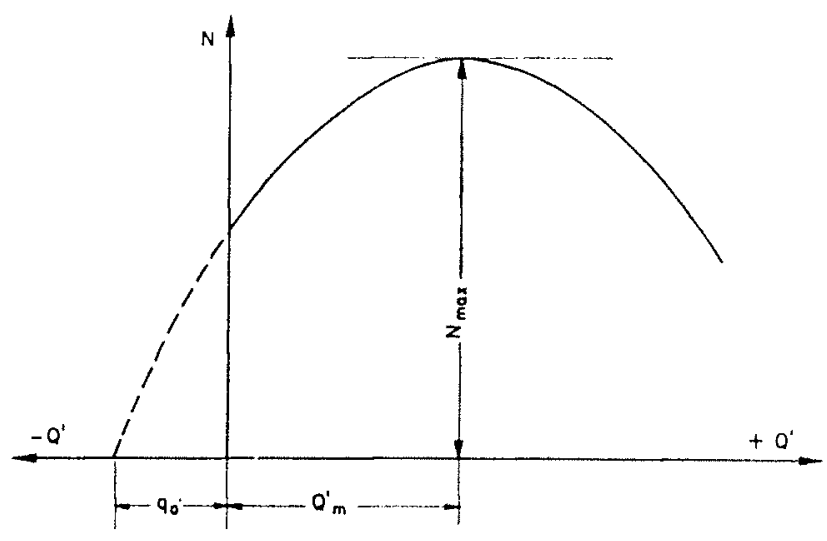

Fic. 2 
du débit conduit à une nouvelle diminution de la puissance, etc.

Cela nous montre que le fonctionnement de la centrale en utilisant des débits $Q_{\mathrm{T}}>Q^{\prime}{ }_{m}+q_{0}$ n'est pas stable. Le sens de l'inégalité (23) est tel qu'elle ne laisse pas s'établir un semblable fonctionnement de la centrale.

Dans la pratique, la condition (23) doit toujours être observée avec une marge de sécurité définie; par exemple le débit maximum de la centrale doit être :

$$
\mathrm{Q}_{\mathrm{TH} \max } \leqq(0,7 \div 0,8)\left(Q_{m}^{\prime}+q_{0}\right)
$$

Il faut remarquer que la condition (25) peut conduire très rarement à des limitations - seulement dans des cas spéciaux; ordinairement, il n'est pas économique d'admettre des pertes de charge si grandes.

Naturellement, les critères (21) et (23) sont également valables dans les cas où $q_{0}=0$, parce que, pendant l'exploitation, on peut attendre, par intervalles, un arrêt du débit supplémentaire.

\section{PREMIER GROUPE DES CAS PARTICULIERS}

1. Supposons que $\mathrm{L}^{\prime \prime \prime}=\mathrm{L}^{\prime \prime \prime}{ }_{r}=0 ; k^{\prime \prime \prime}=0$; cela indique que les deux canaux en charge aboutissent dans la chambre d'équilibre, c'esta-dire que le système hydraulique passe au système montré par la figure 3.

Dans ce cas, l'équation caractéristique sera aussi du quatrième degré et ses coefficients seront :

$$
\begin{aligned}
& \mathrm{A}_{0}=\frac{\mathrm{L}_{r}^{\prime} \cdot \mathrm{L}_{r}^{\prime \prime}}{g} \\
& \mathrm{~A}_{1}=-\frac{\mathrm{Q}_{\mathrm{T}_{0}}}{g \mathrm{H}_{1} \mathrm{~F}} \mathrm{~L}^{\prime}, \mathrm{L}^{\prime \prime}{ }_{r}+2\left(k^{\prime} \mathrm{Q}_{0} \mathrm{~L}^{\prime \prime}{ }_{r}+k^{\prime \prime} \mathrm{Q}^{\prime \prime}{ }_{0} \mathrm{~L}^{\prime}{ }_{r}\right)
\end{aligned}
$$

$$
\begin{aligned}
\mathrm{A}_{2}= & -\frac{2 \mathrm{Q}_{\mathrm{T}_{0}}}{\mathrm{H}_{1} \mathrm{~F}}\left(k^{\prime}{\dot{Q^{\prime}}}_{0} \mathrm{~L}^{\prime \prime}{ }_{r}+k^{\prime \prime} \mathrm{Q}^{\prime \prime}{ }_{0} \mathrm{~L}_{r}^{\prime}\right) \\
& +4 g k^{\prime} \mathrm{Q}_{0}^{\prime} k^{\prime \prime} \mathrm{Q}^{\prime \prime}{ }_{0}+\frac{1}{\mathrm{~F}}\left(\mathrm{~L}_{r}^{\prime}+\mathrm{L}_{r}^{\prime \prime}\right)+\frac{\mathrm{L}_{r}^{\prime}}{f} \\
\mathrm{~A}_{3}= & -4 g \frac{\mathrm{Q}_{\mathrm{T}_{0}}}{\mathrm{H}_{1} \mathrm{~F}}\left(k^{\prime} \mathrm{Q}_{0}^{\prime} k^{\prime \prime} \mathrm{Q}_{{ }_{0}}+\frac{\mathrm{L}_{r}^{\prime}}{4 g f}\right)+ \\
+ & 2 g k^{\prime} \mathrm{Q}_{0}^{\prime}\left(\frac{1}{f}+\frac{1}{\mathrm{~F}}\right)+\frac{2 g k^{\prime \prime} \mathrm{Q}_{0}^{\prime \prime}}{\mathrm{F}} \\
\mathrm{A}_{4}= & \frac{g}{f \mathrm{~F}}\left(1-2 k^{\prime} \mathrm{Q}_{0}^{\prime} \frac{\mathrm{Q}_{\mathrm{T}_{0}}}{\mathrm{H}_{1}}\right) .
\end{aligned}
$$

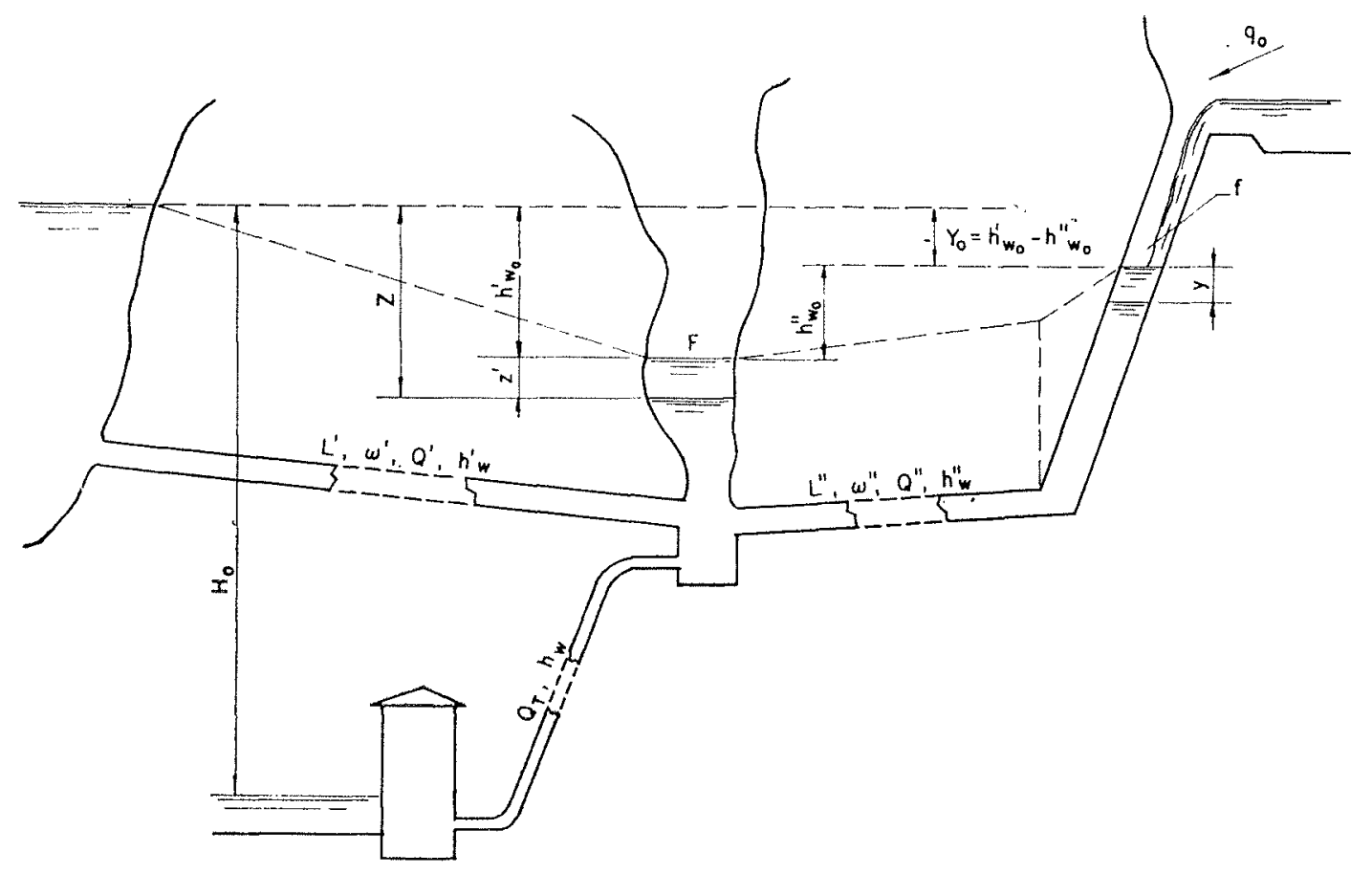

FIG. 3 


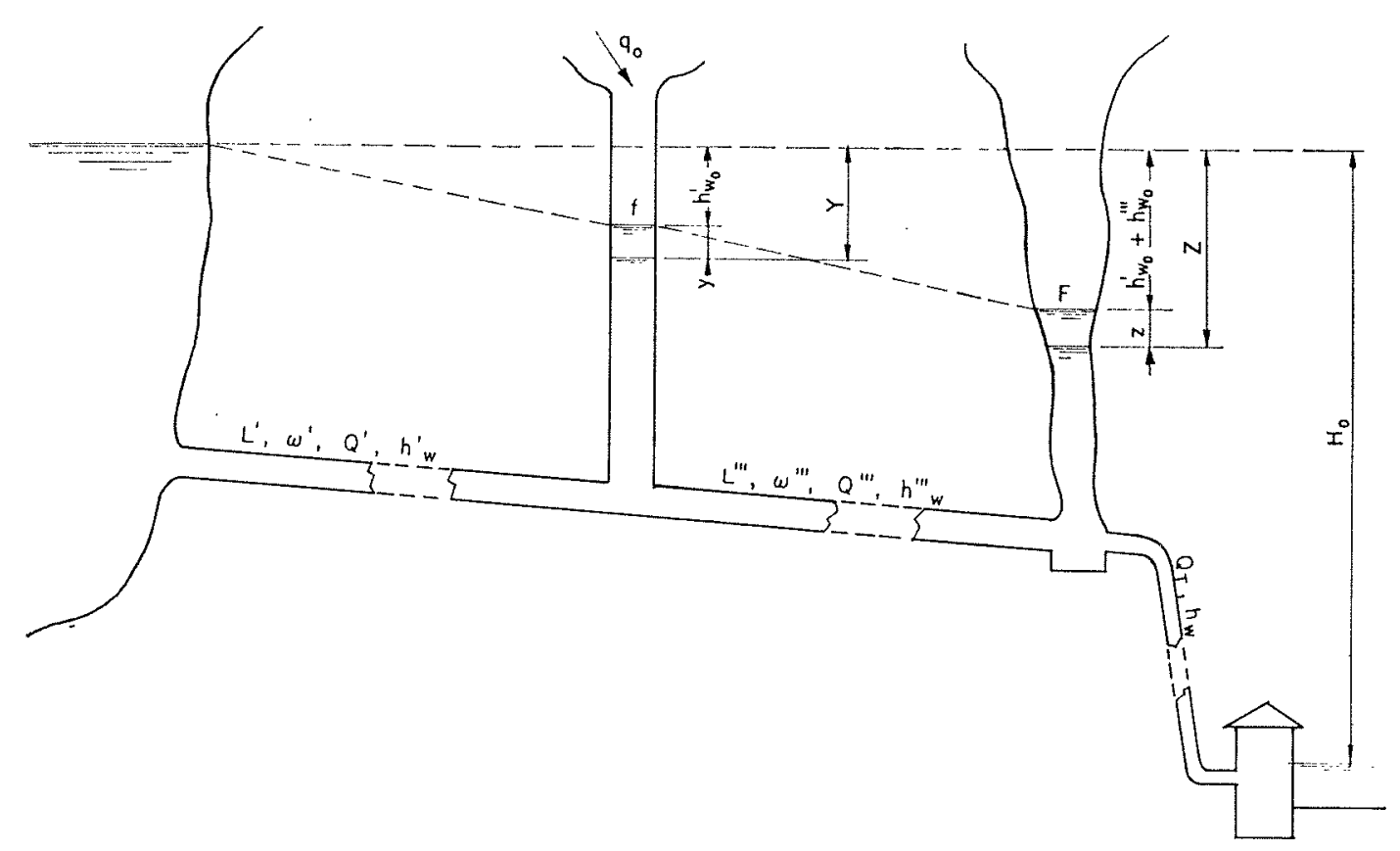

FIG. 4

Les critères de la stabilité hydraulique de la chambre d'équilibre auront la même forme que dans le cas général, notamment :

$$
\mathrm{F}_{\min }>\mathrm{F}_{c r}
$$

où $\mathrm{F}_{c r}$ sera la plus grande racine positive de l'équation :

$$
\mu_{0,0} \mathbf{F}_{c r}{ }^{3}+\mu_{1} \mathbf{F}_{c r}{ }^{2}+u_{2} \mathbf{F}_{c r}+\mu_{3}=0
$$

et :

$$
h_{w_{0}}^{\prime}+h_{w_{0}}<\frac{\mathrm{H}_{0} \cdots-2 k^{\prime} \mathrm{Q}_{0}^{\prime} q_{0}}{3}
$$

2. Si nous posons que $L^{\prime \prime}=L^{\prime \prime}{ }_{r}=0, k^{\prime \prime}=0$ au lieu du système montré par la figure 1, nous obtiendrons le système indiqué par la figure 4 .

L'équation caractéristique est aussi du quaIrième degré avec les coefficients suivants :

$$
\begin{aligned}
\mathrm{A}_{1 \prime}= & \frac{\mathrm{L}_{r}^{\prime} \mathrm{L}^{\prime \prime \prime}{ }_{r}}{g} \\
A_{1}= & -\frac{\mathrm{Q}_{\mathrm{T}_{6}}}{g \mathrm{H}_{1} \mathrm{~F}} \mathrm{~L}_{r}^{\prime} \mathrm{L}^{\prime \prime \prime}{ }_{r}+2\left(k^{\prime} \mathrm{Q}_{0}^{\prime} \mathrm{L}_{{ }^{\prime \prime \prime}}{ }_{r}+k^{\prime \prime \prime} \mathrm{Q}^{\prime \prime \prime}{ }_{0} \mathrm{~L}_{r}^{\prime}\right) \\
\Lambda_{2}= & -\frac{2 \mathrm{Q}_{\mathrm{T}_{0}}}{\mathrm{H}_{1} \mathrm{~F}}\left(k^{\prime} \mathrm{Q}_{0}^{\prime} \mathrm{L}^{\prime \prime \prime}{ }_{r}+k^{\prime \prime \prime} \mathrm{Q}^{\prime \prime \prime}{ }_{0} \mathrm{~L}_{r}^{\prime}\right)+ \\
& +4 g k^{\prime} \mathrm{Q}_{0}^{\prime}{ }_{0} k^{\prime \prime \prime} \mathrm{Q}^{\prime \prime \prime}{ }_{0}+\frac{1}{f}\left(\mathrm{~L}_{r}^{\prime}+\mathrm{L}^{\prime \prime \prime}{ }_{r}\right)+\frac{\mathrm{L}_{r}^{\prime}}{\mathrm{F}}
\end{aligned}
$$

$$
\begin{aligned}
\mathrm{A}_{3}= & -4 g \frac{\mathrm{Q}_{\mathrm{T}_{0}}}{\mathrm{H}_{1} \mathrm{~F}}\left(k^{\prime} \mathrm{Q}_{0}^{\prime} k^{\prime \prime \prime} \mathrm{Q}^{\prime \prime \prime}{ }_{0}+\frac{\mathrm{L}_{r}^{\prime}+\mathrm{L}^{\prime \prime \prime}{ }_{r}}{4 g f}\right)+ \\
& +\frac{2 g}{f}\left(k^{\prime} \mathrm{Q}_{0}^{\prime}+k^{\prime \prime \prime} \mathrm{Q}^{\prime \prime \prime}{ }_{6}\right)+\frac{2 g}{\mathrm{~F}} k^{\prime} \mathbf{Q}_{0}^{\prime} \\
\mathrm{A}_{4}= & \frac{g}{f \mathrm{~F}}\left[1-\frac{2 \mathrm{Q}_{\mathrm{T}_{0}}}{\mathrm{H}_{1}}\left(k^{\prime} \mathrm{Q}_{0}^{\prime}+k^{\prime \prime \prime} \mathrm{Q}^{\prime \prime \prime}{ }_{0}\right)\right]
\end{aligned}
$$

En conséquence, dans ce cas, le système devient un systeme avec une dérivation en charge et deux chambres d'équilibre; dans la chambre moyenne se déverse le débit supplémentaire.

Les conditions de stabilité des phénomènes hydrauliques dans les chambres d'équilibre sont :

$$
\mathbf{F}_{\min }>\mathbf{F}_{c r}
$$

Ici $F_{t r}$ est aussi la plus grande racine de l'érquation algébrique :

$$
\mu_{0,0} \mathrm{~F}_{c r}{ }^{3}+\mu_{1} \mathrm{~F}_{c r}{ }^{2}+\mu_{2} \mathrm{~F}_{c r}+\mu_{3}=0
$$

et :

$$
h_{w_{v}}^{\prime}+h^{\prime \prime \prime} w_{v}+h_{w_{o}}<\frac{\mathrm{H}_{0}-2 h^{\prime} \mathbf{Q}_{0}^{\prime} q_{0}}{3}
$$

3. Supposons que $L^{\prime \prime}=L^{\prime \prime},=0, l^{\prime \prime}=0$, et qu'en outre $f$ soit assez petit pour ètre négligeable; on peut admettre qu'il tend vers zéro: $f \rightarrow 0$. Alors, l'équation caractéristique se transforme en une équation algébrique du deuxiòme degré dont les coefficients sont: 


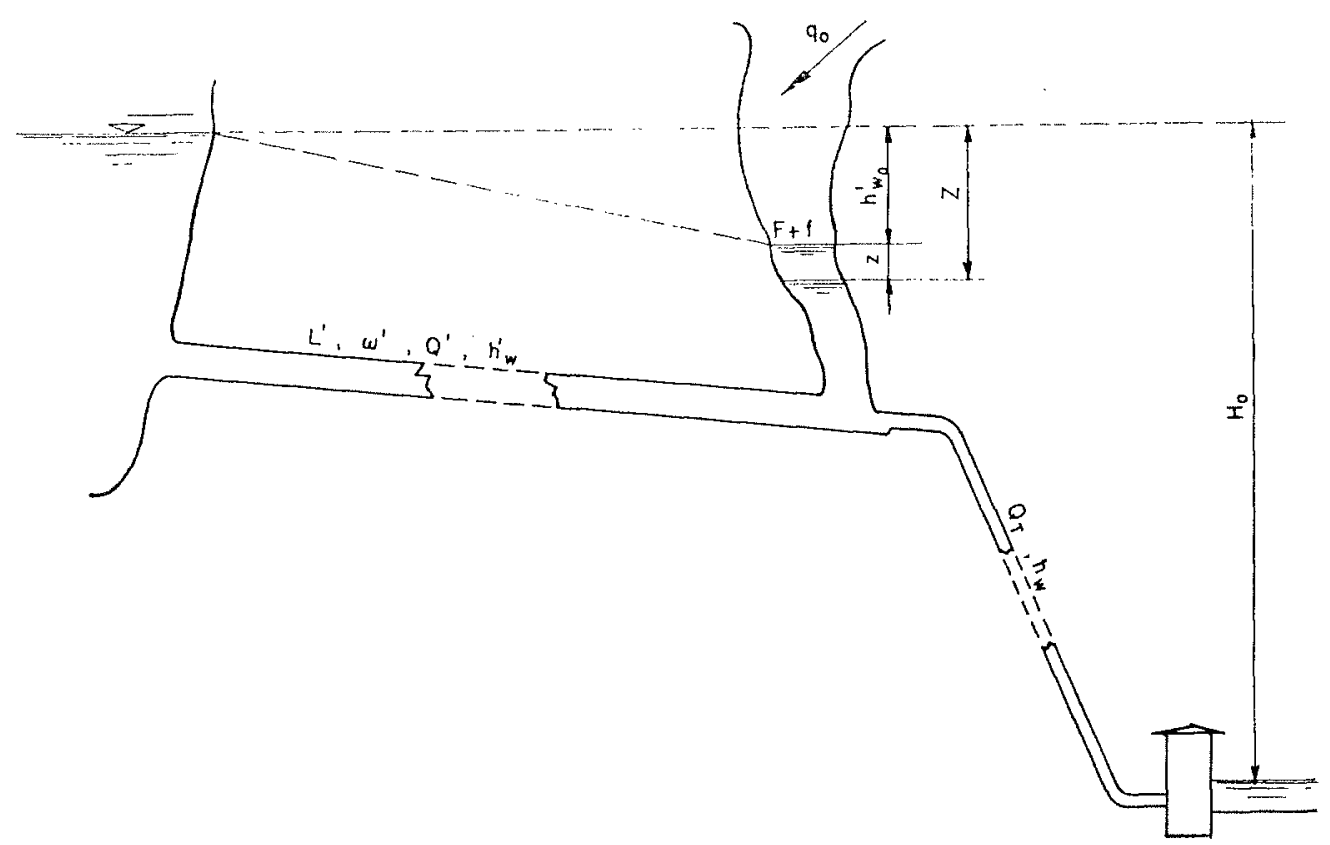

Fig. 5

$$
\begin{aligned}
& \mathrm{A}_{1,}=0 \\
& \mathrm{~A}_{1}=0 \\
& \mathrm{~A}_{\cdot \mathrm{L}}=\mathrm{L}_{r}^{\prime}+\mathrm{L}^{\prime \prime \prime}{ }_{r} \\
& \mathrm{~A}_{3}=-\frac{\mathrm{Q}_{\mathrm{T}_{0}}}{\mathrm{H}_{1} \mathrm{~F}}\left(\mathrm{~L}_{r}^{\prime}+\mathrm{L}^{\prime \prime \prime}{ }_{r}\right)+2 g\left(k^{\prime} \mathrm{Q}_{0}^{\prime}+k^{\prime \prime \prime} \mathrm{Q}^{\prime \prime \prime}{ }_{0}\right) \\
& \mathrm{A}_{4}=\frac{g}{\mathrm{~F}}\left[1-\frac{2 \mathrm{Q}_{\mathrm{T}_{\mathrm{o}}}}{\mathrm{H}_{1}}\left(k^{\prime} \mathrm{Q}_{0}^{\prime}+k^{\prime \prime \prime} \mathrm{Q}^{\prime \prime \prime}{ }_{0}\right)\right]
\end{aligned}
$$

Du point de vue physique, cela signifie que le déversement du débit supplémentaire dans la dérivation en charge s'effectue comme une "injection s uniforme. De cette manière, $\mathrm{Q}^{\prime \prime \prime}=\mathrm{Q}^{\prime}+q_{0}$ et, par conséquent, $d Q^{\prime \prime \prime} / d t=d Q^{\prime} / d t$.

Les conditions de stabilité se réduisent aux conditions suivantes:

$$
\begin{aligned}
& A_{33}>0 \\
& A_{4}>0
\end{aligned}
$$

La première de ces conditions nous donne :

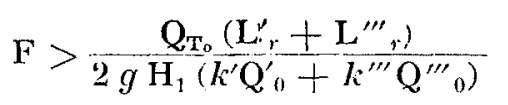

De la deuxième, nous obtiendrons :

$$
h^{\prime}{ }_{0_{0}}+h^{\prime \prime \prime} w_{0}+h_{m_{0}}-\frac{\mathrm{H}_{0}-2 k^{\prime} Q^{\prime}{ }_{0} q_{0}}{3}
$$

Si $q_{n}=0$, des condilions (32) et (33) nous obtiendrons les deux critères de Thoma pour le cas ordinaire d'une chambre d'équilibre, notamment :

$$
\begin{gathered}
\mathbf{F}>\frac{\mathrm{L}_{\gamma}+\mathrm{L}^{\prime \prime \prime}{ }_{r}}{2 g \mathrm{H}_{1}\left(k^{\prime}+k^{\prime \prime \prime}\right)}=\frac{\mathrm{L}_{r}}{2 g k_{\mathrm{r}} \mathrm{H}_{1}} \\
h_{w_{0}}^{\prime}+h^{\prime \prime \prime} w_{0}+h_{w_{0}}=\mathrm{H}_{w_{0}}^{\prime}+h_{w_{0}}<\frac{\mathrm{H}_{0}}{3}
\end{gathered}
$$

Ici, les symboles supplémentaires $\mathrm{L}_{r}$ et $k_{\mathrm{r}}$ ont pour valeurs :

$$
\begin{aligned}
& \mathrm{L}_{r}=\mathrm{L}_{r}{ }_{r}+\mathrm{L}^{\prime \prime \prime}{ }_{r} \\
& \mathrm{k}_{\mathrm{T}}=k^{\prime}+k^{\prime \prime \prime}
\end{aligned}
$$

4. Dans ce cas-ci nous poserons : $\mathrm{L}^{\prime \prime}=\mathrm{L}^{\prime \prime}=0$, $k^{\prime \prime}=0, \mathrm{~L}^{\prime \prime \prime}=\mathrm{L}^{\prime \prime}{ }_{r}=0, k^{\prime \prime \prime}=0$, avec lesquels le système se transforme complètement en un système ordinaire, avec cette seule différence que la chambre d'équilibre admet un débit supplémentaire $q_{0}$ (fig, 5).

Les coefficients de l'égualion caractiristique seront :

$$
\begin{aligned}
& A_{0}=0 \\
& A_{1}=0
\end{aligned}
$$

$$
\begin{aligned}
& A_{2}=L_{r}^{\prime}\left(\frac{1}{\mathrm{~F}}+\frac{1}{f}\right) \\
& A_{3}=-\frac{\mathrm{Q}_{\mathrm{r}_{0}} \mathrm{~L}_{r}^{\prime}}{\mathrm{H}_{1} \mathrm{~F} f}+2 g k^{\prime} \mathrm{Q}_{0}^{\prime}\left(\frac{1}{\mathrm{~F}}+\frac{1}{f}\right)
\end{aligned}
$$


$\mathrm{A}_{4}=\frac{g}{\mathrm{~F} f}\left(1-2 k^{\prime} \mathrm{Q}_{0}^{\prime} \frac{\mathrm{Q}_{\mathrm{T}_{0}}}{\mathrm{H}_{1}}\right)$

c'est-à-dire que les conditions de stabilité se réduisent aux conditions suivantes :

$$
\begin{aligned}
& A_{3}>0 \\
& A_{4}>0
\end{aligned}
$$

De ces conditions, nous obtiendrons :

$$
\begin{gathered}
\mathrm{F}+f>\frac{\mathrm{L}_{r}^{\prime}}{2 g k^{\prime} \mathrm{H}_{1}}\left(1+\frac{\mathcal{q}_{0}}{\mathrm{Q}_{0}^{\prime}}\right) \\
h_{t_{0,}^{\prime}}+h_{u_{0}}<\frac{\mathrm{H}_{0}-2 k^{\prime} \mathrm{Q}_{0}^{\prime} q_{0}}{3}
\end{gathered}
$$

Il faut remarquer que, selon nos suppositions, le puits et la chambre d'équilibre se réunissent ct que la somme de leurs sections forme la section totale de la chambre d'équilibre :

$$
\mathbf{F}_{\mathrm{r}}=\mathbf{F}+f .
$$

Examinons d'une manière un peu plus détaillée les conditions (38) et (39).

$\mathrm{Si}$, à l'instant initial, la centrale a fonctionné avec un débit $\mathrm{Q}_{\mathrm{T}_{0}}=2 q_{0}$, c'est-à-dire si $\mathrm{Q}_{n}^{\prime}=q_{0}$, nous aurons :

$$
\mathrm{F}+f>\frac{\mathrm{L}_{r}^{\prime}}{g k^{\prime} \mathrm{H}_{1}}
$$

et :

$$
5 h_{w_{0}}^{\prime}+3 h_{w_{0}}<\mathrm{H}_{0}
$$

L'inégalité (40) montre qu'il est nécessaire d'avoir une section à peu près deux fois plus grande que celle donnée par la formule de Thoma pour que les mouvements du plan d'eau dans la chambre d'équilibre soient stables.

Supposons donc que, dans l'instant initial, la centrale a fonctionné seulement avec le débit supplémentaire $q_{0}$. Cela signifie que le débit prélevé dans la retenue $Q_{0}^{\prime}=0$ et les inćgalités $(36)$ el (37) respectivement (38) et (39) donnent;

$$
\begin{aligned}
& \mathbf{F}+f>\infty \\
& h_{e_{0}}<\frac{\mathrm{H}_{0}}{3}
\end{aligned}
$$

Ici, le symbole « $\infty \gg$ doit être compris comme une grande section de la chambre d'équilibre qui devient aussi une retenue.

A la fin, supposons que $q_{0}=0$.

Alor's :

$$
\begin{aligned}
& F+f>\frac{\mathrm{L}_{r}^{\prime}}{2 g k^{\prime} \mathrm{H}_{1}} \\
& h_{w_{0}}^{\prime}+h_{w_{0}}<\frac{\mathrm{H}_{0}}{3}
\end{aligned}
$$

On voit facilement que les inégalités (44) et (45) représentent les deux critères de Thoma.

En effet, les suppositions précédentes rendent le système complètement identique aux cas ordinaires.

\section{DEUXIEME GROUPE DES CAS PARTICULIERS}

1. Considérons d'abord le cas obtenu en supposant que $f \rightarrow \infty$, c'est-à-dire qu'au lieu du puits, nous aurons un autre lac, A cette supposition correspond le système indiqué par la figure 6.

Pour ce cas particulier, les coefficients de l'équation caractéristique auront les valeurs suivintes:

$$
\begin{aligned}
& A_{0}=\frac{L_{r} L_{L^{\prime \prime \prime}}{ }_{r}+L^{\prime \prime}{ }_{r}\left(L_{r}^{\prime}+L^{\prime \prime \prime}{ }_{r}\right)}{g} \\
& \mathrm{~A}_{1}=-\frac{\mathrm{Q}_{\mathrm{Y}_{n}}}{g \mathrm{H}_{1} \mathrm{~F}}\left[\mathrm{~L}_{r}^{\prime} \mathrm{L}^{\prime \prime \prime}{ }_{r}+\mathrm{L}^{\prime \prime}{ }_{r}\left(\mathrm{~L}_{r}^{\prime}+\mathrm{L}^{\prime \prime \prime}{ }_{r}\right)\right]+ \\
& +2\left[k^{\prime} \mathrm{Q}_{0}^{\prime}\left(\mathrm{L}^{\prime \prime},{ }_{r}+\mathrm{L}^{\prime \prime \prime}{ }_{r}\right)+\right. \\
& \left.+k^{\prime \prime} Q^{\prime \prime}{ }_{0}\left(L_{r}^{\prime}+L^{\prime \prime \prime}{ }_{r}\right)+k^{\prime \prime \prime} Q^{\prime \prime \prime}{ }_{0}\left(L_{r}^{\prime}+L^{\prime \prime}{ }_{r}\right)\right]
\end{aligned}
$$

$$
\begin{aligned}
& \mathrm{A}_{2}=-\frac{2 \mathrm{Q}_{\mathrm{T}_{0}}}{\mathrm{H}_{1} \mathrm{~F}} \cdot k^{\prime} \mathrm{Q}_{n}^{\prime}\left(\mathrm{L}^{\prime \prime}{ }_{r}+\mathrm{L}^{\prime \prime \prime}{ }_{r}\right)+ \\
& \left.+k^{\prime \prime} \mathrm{Q}^{\prime \prime}{ }_{0}\left(\mathrm{~L}_{r}^{\prime}+\mathrm{L}^{\prime \prime \prime}{ }_{r}\right)+k^{\prime \prime \prime} \mathrm{Q}^{\prime \prime \prime}{ }_{0}\left(\mathrm{~L}_{r}^{\prime}+\mathrm{L}_{r}{ }_{r}\right)\right]+ \\
& +4 g\left(k^{\prime} \mathrm{Q}_{0}^{\prime} k^{\prime \prime} \mathrm{Q}^{\prime \prime}{ }_{0}+\left(k^{\prime} \mathrm{Q}_{0}^{\prime} k^{\prime \prime \prime} \mathrm{Q}^{\prime \prime \prime}{ }_{01}+k^{\prime} \mathrm{Q}^{\prime \prime}{ }_{0} k^{\prime \prime \prime} \mathrm{Q}^{\prime \prime \prime}{ }_{0}\right)\right. \\
& +\frac{1}{\mathrm{~F}}\left(\mathrm{~L}_{r}^{\prime}+\mathrm{L}_{\prime^{\prime \prime}}\right) \\
& \mathrm{A}_{3}=-4 g \frac{\mathrm{Q}_{\mathrm{T}_{10}}}{\mathrm{H}_{1} \mathrm{~F}}\left(k^{\prime} \mathbf{Q}_{0}^{\prime} k^{\prime \prime} Q^{\prime \prime}{ }_{0}+k^{\prime} \mathrm{Q}_{0}^{\prime} k^{\prime \prime \prime} \mathrm{Q}^{\prime \prime \prime}{ }_{0}+\right. \\
& \left.+k^{\prime \prime} Q_{0}^{\prime \prime} k^{\prime \prime \prime} Q^{\prime \prime \prime}{ }_{0}\right)+\frac{2 g}{\mathrm{~F}} \cdot\left(k^{\prime} \mathrm{Q}_{0}^{\prime}+k^{\prime \prime} \mathrm{Q}_{0}^{\prime \prime}\right) \\
& \mathrm{A}_{4}=0 .
\end{aligned}
$$




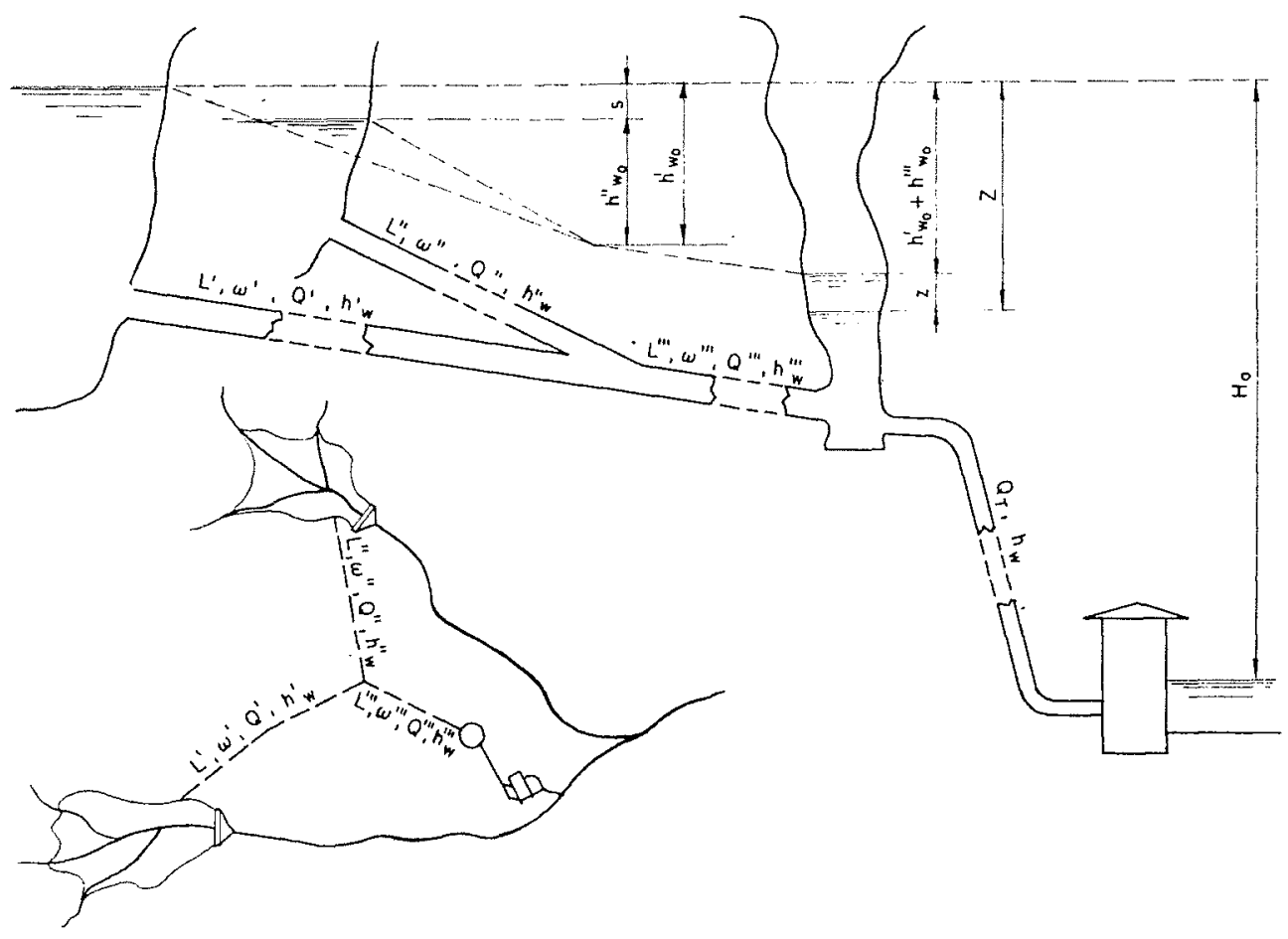

Fig. 6

Une des racines de l'écuation caractéristique aura la valeur triviale $r=0$ et en effet nous aurons une équation algébrique du troisième degré.

Les conditions de stabilité seront :

$$
\begin{aligned}
& A_{3}>0 \\
& A_{1} A_{2}-A_{0} A_{3}>0
\end{aligned}
$$

En remarquant que :

$$
\begin{aligned}
& A_{1}=\frac{\alpha_{1}}{F}+\beta_{1} \\
& A_{2}=\frac{\alpha_{2}}{F}+\beta_{2} \\
& A_{3}=\frac{\alpha_{3}}{F}
\end{aligned}
$$

par rapport a la valeur critique de la section $F$, nous obtiendrons une équation algébrique du deuxième degré, notamment :

$$
\beta_{1} \beta_{2} F_{c r}{ }^{2}+\left(\alpha_{1} \beta_{2}+\beta_{1} \alpha_{2}-A_{0} \alpha_{3}\right) F_{c r}+\alpha_{1} \alpha_{.2}=0
$$

La condition (47) signifie que :

$$
\mathrm{F}_{\min }>\mathrm{F}_{t r}
$$

et la condition (46) :

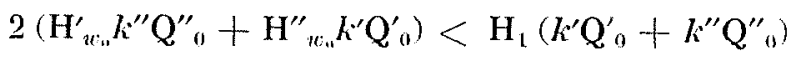

où, entre les pertes de charge, existe la relation :

$$
\mathbf{H}_{w_{0}}^{\prime}=\mathbf{H}_{w_{0}}^{\prime \prime}+s
$$

$s$ est la différence entre les plans d'eau dans les deux lacs.

2. Nous aurons un autre cas particulier en supposant que :

$$
f \rightarrow \infty, \mathrm{L}^{\prime \prime \prime}=\mathrm{L}^{\prime \prime \prime}{ }_{r}=k^{\prime \prime \prime}=0
$$

Les suppositions précédentes correspondent à un système montré par la figure 7 .

Lès coefficients de l'équation caractéristique, dans ce cas sont:

$$
A_{0}=\frac{\mathrm{L}_{r}^{\prime} \cdot \mathrm{L}^{\prime \prime}, r}{g}
$$

$$
\begin{aligned}
& \mathrm{A}_{1}=-\frac{\mathrm{Q}_{\mathrm{T}_{0}}}{g \mathrm{H}_{1} \mathrm{~F}} \mathrm{~L}_{{ }_{r}} \mathrm{~L}^{\prime \prime}{ }_{,}+2\left(k^{\prime} \mathrm{Q}_{01}^{\prime} \mathrm{L}^{\prime \prime}{ }_{r}+k^{\prime \prime} \mathrm{Q}^{\prime \prime}{ }_{0} \mathrm{~L}_{\gamma}^{\prime}\right) \\
& \mathrm{A}_{2}=-\frac{2 \mathrm{Q}_{\mathrm{T}_{0}}}{\mathrm{H}_{1} \mathrm{~F}}\left(K^{\prime} \mathrm{Q}_{0}^{\prime} \mathrm{L}_{2,}{ }_{r}+k^{\prime \prime} \mathrm{Q}^{\prime \prime}{ }_{0} \mathrm{~L}_{{ }^{\prime}}\right)+ \\
& +4 g k^{\prime} \mathrm{Q}^{\prime}{ }^{\prime} k^{\prime \prime} \mathrm{Q}^{\prime \prime}{ }_{n}+\frac{1}{\mathrm{~F}}\left(\mathrm{~L}_{r}^{\prime}+\mathrm{L}_{{ }_{r}}{ }^{\prime}\right)
\end{aligned}
$$

$\mathrm{A}_{3}=-4 g \frac{\mathrm{Q}_{\mathrm{T}_{1}}}{\mathrm{H}_{1} \mathrm{~F}} k^{\prime} \mathrm{Q}_{0}^{\prime} k^{\prime \prime} \mathrm{Q}^{\prime \prime}{ }_{0}+\frac{2 g}{\mathrm{~F}}\left(k^{\prime} \mathrm{Q}_{0}^{\prime}+k^{\prime \prime} \mathrm{Q}^{\prime \prime}{ }_{0}\right)$

$$
A_{4}=0
$$




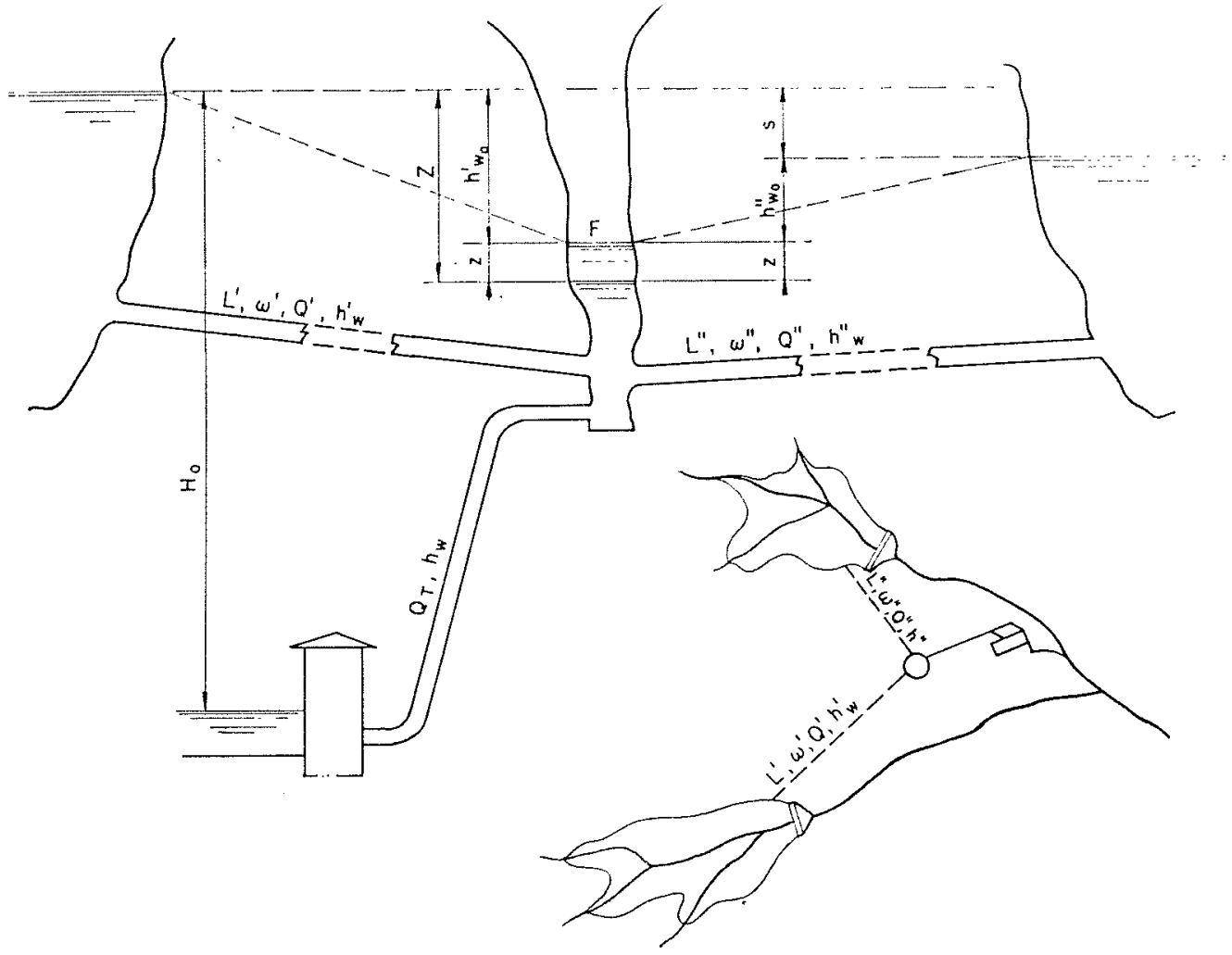

FIG. 7

Pour que les phénomènes hydrauliques soient slables, il est nécessaire et suffisant d'observer les conditions (47) et (46) qui conduisent aux inçgalites :

$$
\mathrm{F}_{\text {min }}>\mathrm{F}_{c r}
$$

$2\left(h^{\prime}{ }_{w_{0}} k^{\prime \prime} \mathrm{Q}^{\prime \prime}{ }_{0}+h^{\prime \prime}{ }_{w_{0}} k^{\prime} \mathrm{Q}_{0}^{\prime}\right)<\mathrm{H}_{1}\left(k^{\prime} Q^{\prime}{ }_{0}+k^{\prime \prime} \mathrm{Q}^{\prime \prime}{ }_{0}\right)$

Sì $s=0$, au lieu de (49) et (51), nous aturons les conditions plus simples suivantes:

$$
\begin{gathered}
\mathrm{H}_{w_{0}}+h_{w_{0}}<\frac{\mathrm{H}_{0}}{3} \\
h_{w_{0}}^{\prime}+h_{w_{0}}<\frac{\mathrm{H}_{0}}{3}
\end{gathered}
$$

Finalenent, il faut remarquer que l'ctude est effectuée sans prendre en considération la variation du rendement $\eta$ des groupes hydroélectriques avec la variation de la puissance, et aussi ce fait que les centrales modernes fonctionnent dans un réseau électrique interconnecté el que la charge se répartit entre toutes les centrales du réseau.

\section{BIBLIOGRAPHIE}

1. Lscinoe (L.) a Goutrin (V.). Comparaison de diverses méthodes de calcul appliquées à la chambre d'écuilibre complexe de l'usine de Bioge. La Houlle Blanche, $\mathrm{n}^{\circ} 3,1948$.

2. Momosov (A. A.). Utilisation de lénergie hydraulique. Gosénergoisdat, Moscou-Lenïngrad, 1948 (en russe).

3. Kantrelischyul (N. A.). Stabilité hydraulique des chambres d'équilibre.

Izvestija V.N.I.I.G., tome 26, Gosénergoisdal, Leningrad, 1940 (en russe).

4. Voct (Fr.). Berechnung und honstruktion des Wasserschlosses.

Verlag von Ferdinand Enke, Stutgart, 1923.
5. Evangelist (Giuseppe). Sopra la stabilita dei sistemi complessi di gallerie in pressione e pozzi piezometrici.

L'Energia Elettrica, 10" 1, 1955.

6. Kä̈rev (P.). Conditions de stabilité des oscillation dans les chambres d'équilibre des usines hydroélectriques collectant un débit supplémentaire.

Revue Hidrotelhnika $i$ Melioracii, $n^{\circ} 4$, 1959, Sofia (en bulgare).

7. Kä̈chev (P.) et VAssilev (A.). De l'action hydraulique des chambres d'équilibre dans certains cas compliqués d'utilisation de l'énergie hydraulique.

Ouvrages de l'Institut des recherches scientifiques d'électrification, livre II, Izdatelstvo Tekhnika, Sofia, 1959 (en bulgare). 